\title{
High-temperature reaction of sodium vapour with quartz glass
}

\author{
Radovan Nosek 1,*, Zuzana Kolkova ${ }^{2}$, Libor Trško ${ }^{2}$, Tatiana Liptáková3 ${ }^{3}$ Jan Procházka ${ }^{4}$, \\ Ondřej Libera ${ }^{4}$, and Petra Bublíková ${ }^{4}$ \\ ${ }^{1}$ University of Žilina, Faculty of Mechanical Engineering, Department of Power Engineering, \\ Univerzitná 8215/1, 01026 Žilina, Slovakia \\ ${ }^{2}$ Research Centre of the University of Žilina, Univerzitná 8215/1, 01026 Žilina, Slovakia \\ ${ }^{3}$ University of Žilina, Faculty of Mechanical Engineering, Department of Material Engineering, \\ Univerzitná 8215/1, 01026 Žilina, Slovakia \\ ${ }^{4}$ Research Centre Řež, Hlavní 130, 25068 Husinec, Czech Republic
}

\begin{abstract}
Heat pipes are one of the most efficient ways to transfer thermal energy hundred times more efficiently than copper. In this work, we present the investigation of sodium terminal velocity inside a glass pulsating heat pipe. Velocity measurements were conducted under operating conditions within the range of $500-1100{ }^{\circ} \mathrm{C}$. Since sodium is generally able to etch glass and ceramic materials, its presence resulted in glass reduction and sodium oxidation. From the XPS analysis of specimens extracted from a glass pipe after sodium explosion, it can be concluded that the reaction products are sodium oxide $\mathrm{Na}_{2} \mathrm{O}$ and a thin layer of carbon, which is localized on the $\mathrm{SiO}_{2}$ glass. The character of damage induced by chemical reactions is analysed in this manuscript.
\end{abstract}

\section{Introduction}

Primary aluminium is the material obtained from electrolytic cells or pots during an electrolytic reduction of metallurgical alumina (aluminium oxide). Therefore, it excludes alloying additives and recycled aluminium. The electrolytic cell uses electrolysis to produce aluminium in metal form. It means, that large currents must be used in order to refine the aluminium oxide to the pure aluminium. Unequal current distribution inside aluminium electrolytic cells causes energy losses, a phenomena impacting both the economy and the environment. Around $50 \%$ of the supplied energy is the lost as thermal waste. The heat losses are necessary to maintain a frozen side ledge to protect the side walls of cells. As the energy costs account for $40 \%$ of the total production costs, it is clear that the heat recovery optimization offers a significant economical end environmental potential in the industry.

Although waste heat is abundant in primary production plants, possibilities of thermal integration remain limited. Some of the main reasons impeding waste heat recovery and thermal integration in smelters are the low quality of the waste heat (i.e., low temperature)

* Corresponding author: radovan.nosek@,fstroj.uniza.sk 
and large distances between heat sources and locations where heat could be used (herein called heat sinks). Extracting the waste heat (capturing it in a heat transfer fluid) also requires adapted heat-exchanger designs. The technology based on the use of heat pipes has been developed for utilization of energy from the waste heat produced in the electrolytic process. The heat recovery system can control the ledge thickness and thermal energy can be utilized by other energy demanding processes while increasing the aluminium production by $20 \%$ and still achieve thermal balance. With the heat recovery system, 10 $12 \%$ of the total energy consumed during the process can be recovered [1].

The project aims was to develop an innovative heat-to-electricity converter without any moving parts, placed on the pulsating heat pipe (PHP). The device can generate electric power by flowing fluid interacting with the magnetic field. The project aim was to investigate the fluid velocity within the heat pipe during the flowing.

\section{Technology of efficient energy utilization}

The investigated technology for the energy utilization of waste heat consists of PHP, which is a two phase heat transfer device with outstanding effective thermal conductivity. It is a vacuum tight device consisting of an envelope (here made of quartz glass) and flowing fluid (liquid sodium), which transfers heat under isothermal conditions at vaporization [2]. The fluid used in this study can operate in the range of $500-1100{ }^{\circ} \mathrm{C}$. Due to its electron configuration with a single electron in the valence shell, sodium is a very reactive element with one of the lowest electronegativity. It was expected, that its high chemical reactivity will be mitigated by high vacuum [3]. The evaporation part of the PHP was heated up to $750{ }^{\circ} \mathrm{C}$ which resulted in immediate explosion of the device (Figure 1).

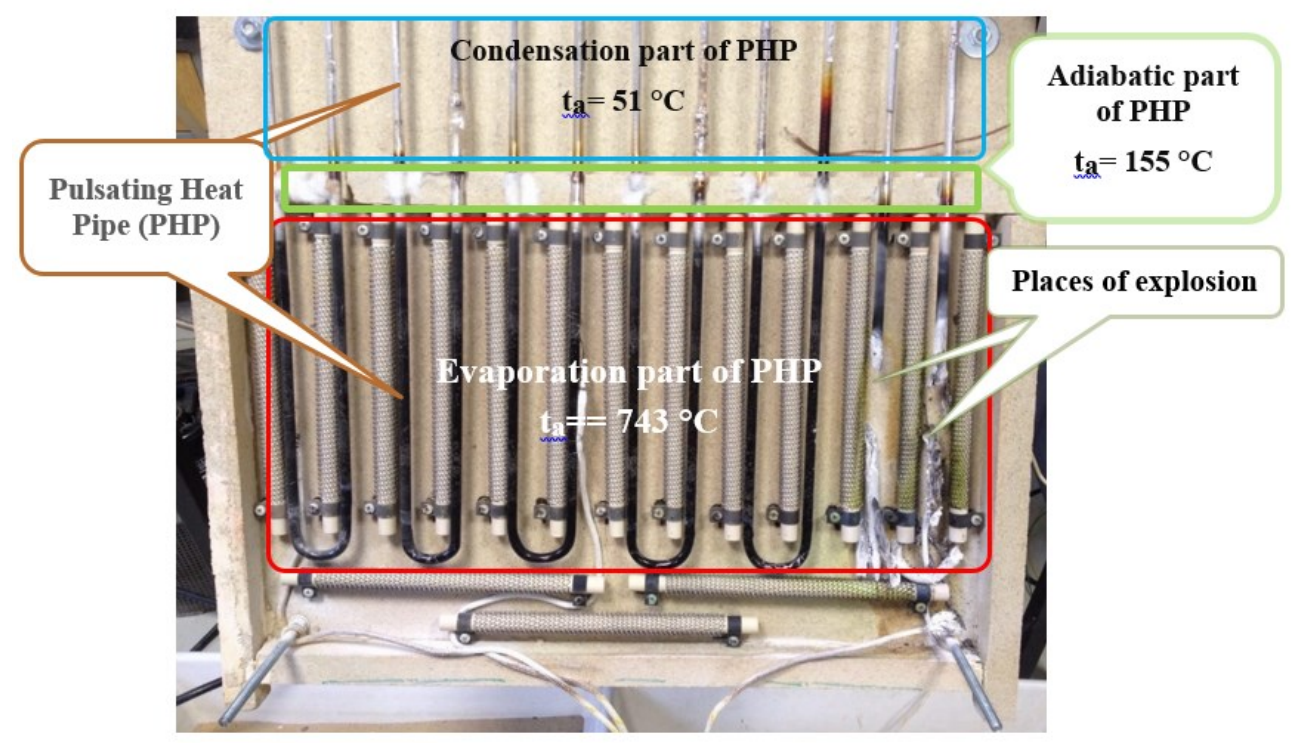

Fig. 1. Detail of the PHP experimental setup and the damage caused by explosion.

It is visible that the character of compounds inside the PHP changes over different parts (condensation - adiabatic transfer - evaporation) is caused by different temperatures. The average temperature $\left(t_{a}\right)$ in each PHP part is shown in Figure 1. For the identification of the black substance covering the pipe walls, the Scanning Electron Microscopy (SEM) equipped with Energy Dispersive X-Ray Spectroscopy (EDS) and X-Ray photoelectron 
spectroscopy (XPS) methods were used to analyse the factors causing the explosion. The XPS analysis shows the presence of pure carbon in high concentration upon the quartz pipe wall (Table 1.).

Table 1. Chemical composition of the black products on the pipe wall, XPS analysis.

\begin{tabular}{|c|c|c|}
\hline Element & Weight \% & Atomic \% \\
\hline Carbon C & 81.11 & 87.79 \\
\hline Oxygen O & 9.9 & 8.04 \\
\hline Silicone Si & 9.0 & 4.17 \\
\hline
\end{tabular}

Based on the XPS results, the explosion was caused by the high temperature followed by the devitrification of fused silica pipe due to sodium [4] and heterogeneity of reactions inside the experimental system. Increasing of sodium diffusion through the weakened walls and the structural failure were followed by chemical reaction of sodium with components of ambient atmosphere.

Under the thermal load, the devitrified areas tend to weaken the PHP and change its mechanical properties. Also, in such devitrified areas the crystallization of previous amorphous structure was observed. That was probably caused by diffusion channels for transfer of environmental contaminants into the molten sodium [5]. The mechanism of devitrification enhanced by sodium, involves diffusion of sodium into the fused quartz. The sodium is assumed to diffuse quickly through the open structure of the silica via strings of interconnected interstices [6]. The sodium sets up nucleation sites for crystallization. One can imagine a piece of fractured glass pipe from a wet oxidation has failed due to devitrification. It can be observed, that the heavily devitrified region is inside of the PHP wall. It can be caused by increasing rate of devitrification from water [7].

Furthermore, the SEM analysis of the damaged PHP exposed to the molten sodium at four different temperatures were carried out (Figure 2).

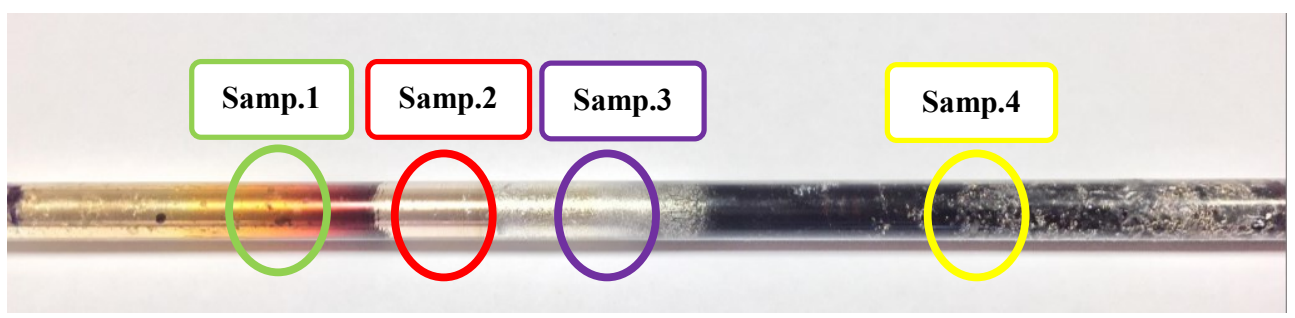

Fig. 2. Labelling of analysed areas of the exposed PHP.

For the SEM analyses, the quartz samples were mechanically cleaned of surface deposits and subsequently washed in water. The samples were mounted using the colloidal silver and sputtered by $20 \mathrm{~nm}$ thick carbon film, to ensure charge removal. The results of SEM (EDS) analyses (chemical mapping and point analyses) of all samples are shown in Figure 3-14. The sample areas were imaged in Secondary (SE) and Backscattered (BSE) electrons. For the disambiguation, the spectral lines are vertically shifted. 


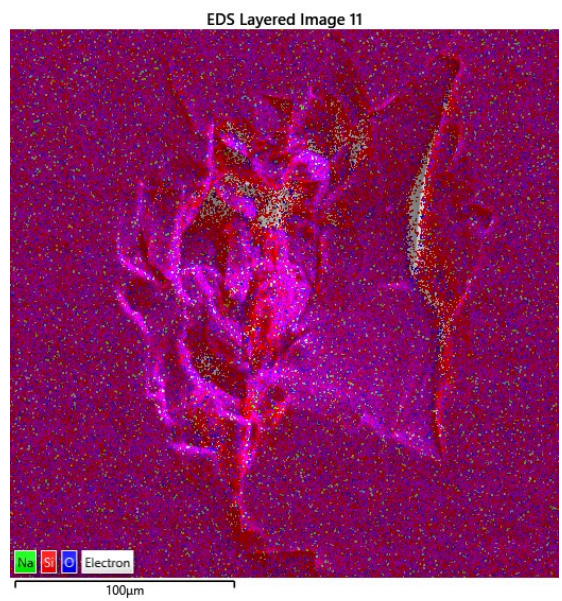

Fig. 3. Sample 1 - EDS mapping of sodium (green channel), silicon (red channel) and oxygen (blue channel) elements.

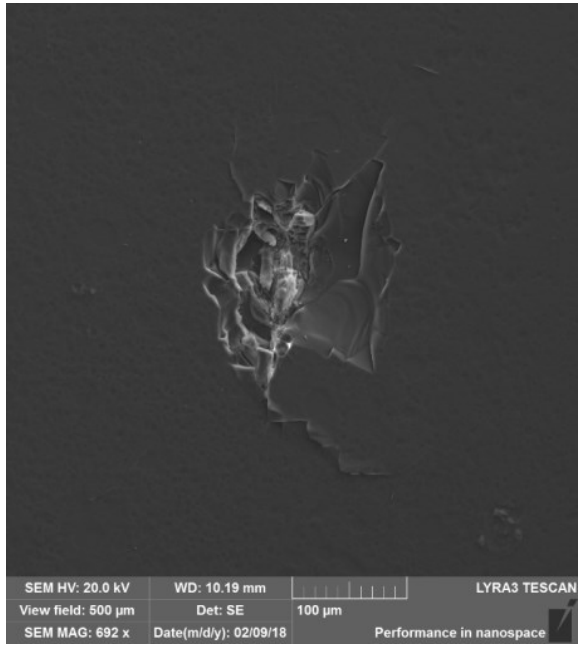

a)

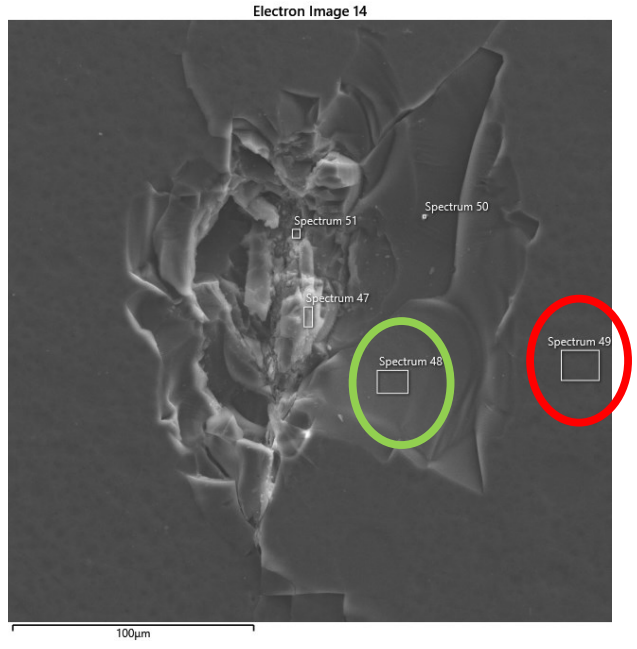

b)

Fig. 4. Sample 1 - SEM imaging of the inner surface (a). Detailed SEM image of the crack in the inner surface with analysed regions emphasized (b).

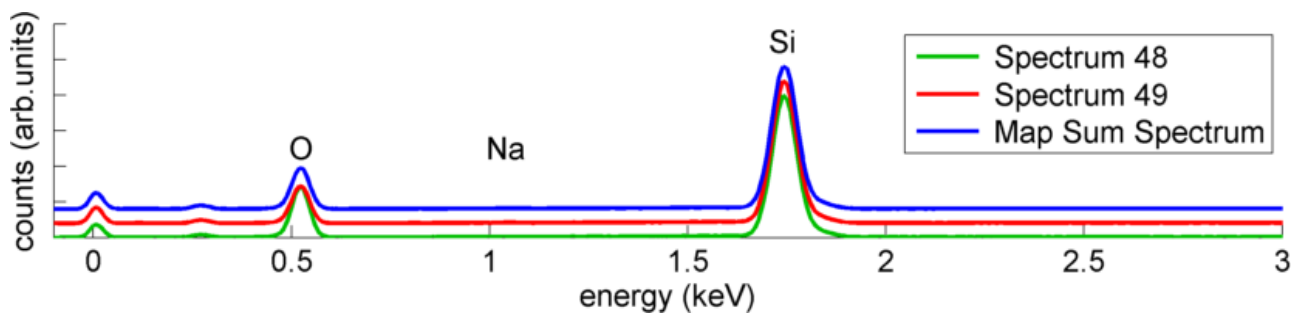

Fig. 5. Sample 1 - EDS spectrum of the region in Fig. 4a (blue line), the sodium-exposed surface (red line) and the uncovered surface (green line). 


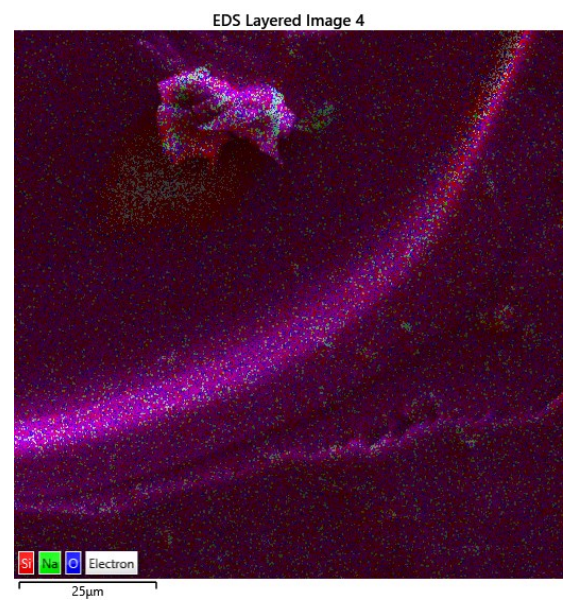

Fig. 6. Sample 3 - EDS element mapping.

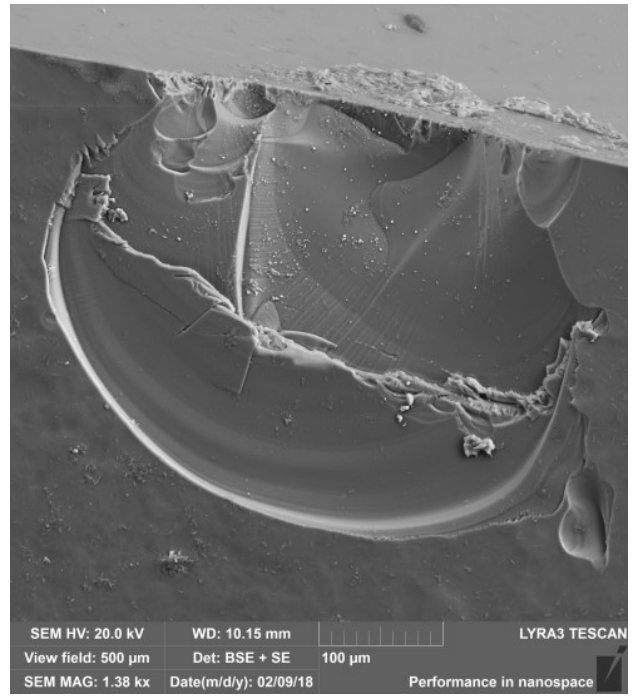

a)

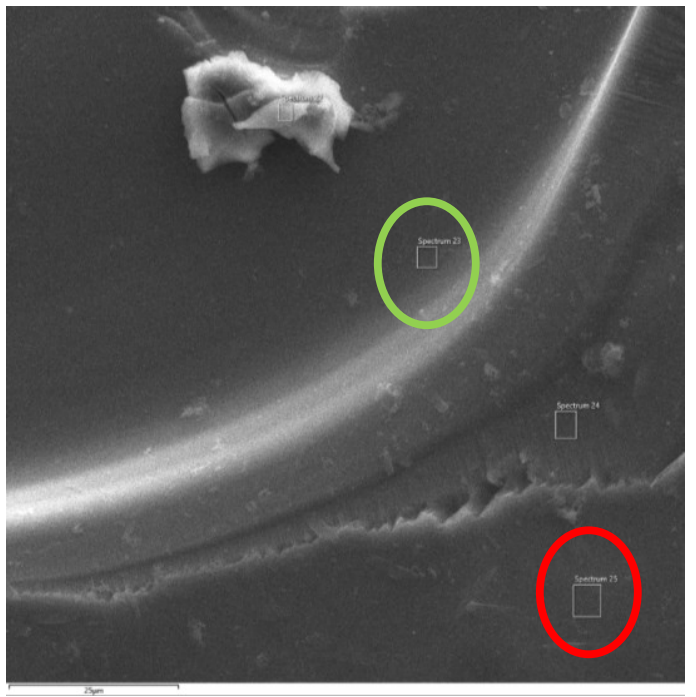

b)

Fig. 7. Sample 2 - SEM imaging of sample surface (a) and crack detail with labelled areas of EDS analysis (b).

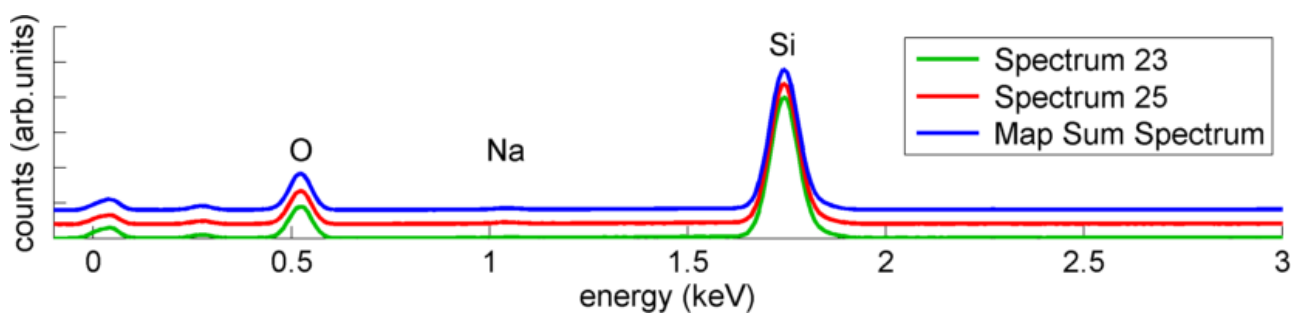

Fig. 8. Sample 2 - EDS spectrum of selected areas of the exposed material (Spectrum 23), the inner surface of the PHP (Spectrum 25) and EDS spectrum from the whole image (Map Sum Spectrum). 


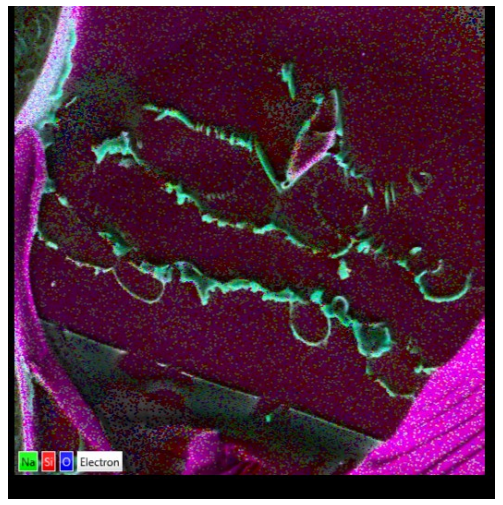

Fig. 9. Sample 2 - EDS element mapping.

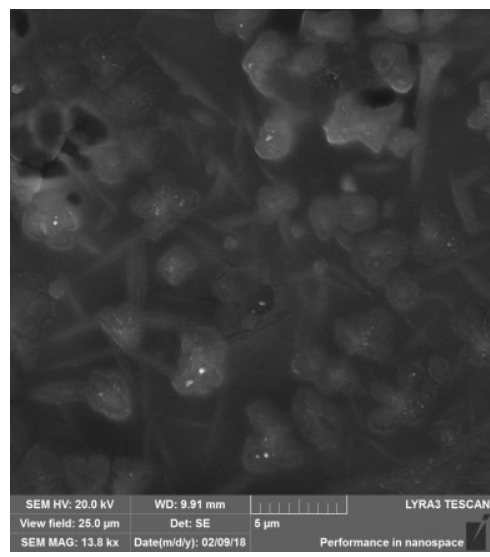

a)

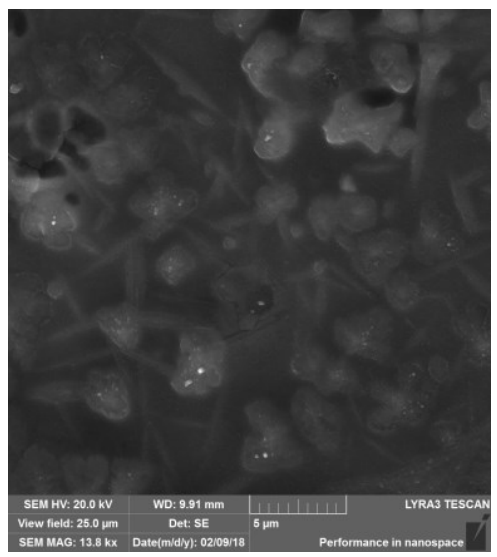

b)

Fig. 10. Sample 3 - Detailed image of high sodium concentration.

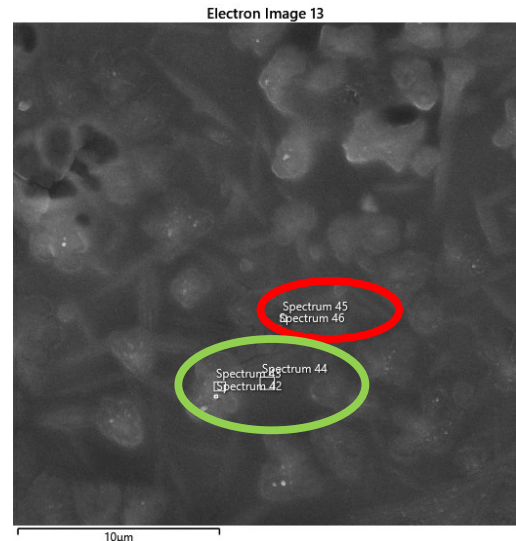

a)

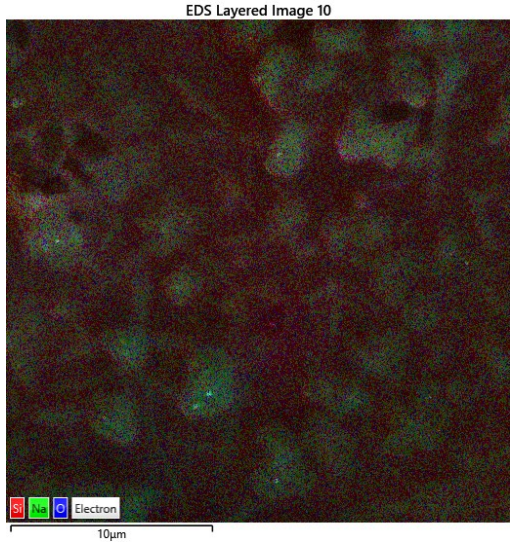

b)

Fig. 11. Sample 3 - Detailed image of high sodium concentration with marked areas of EDS analysis. 


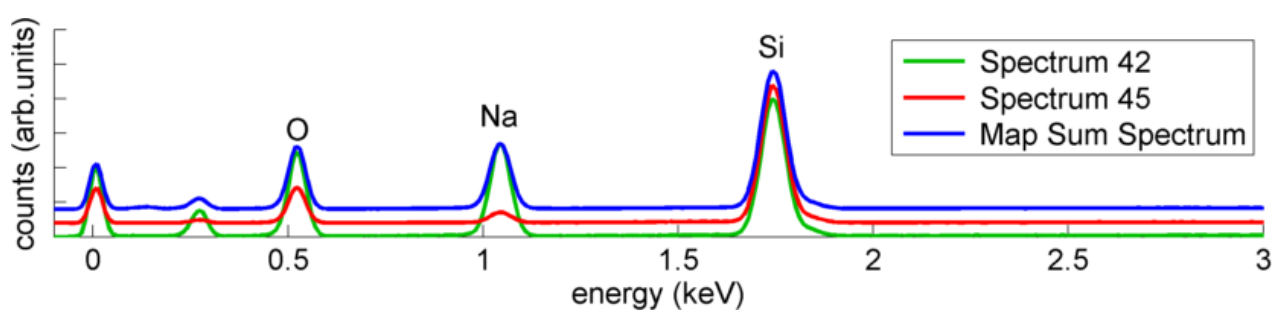

Fig. 12. Sample 3 - EDS spectra of selected areas of exposed material (Spectrum 42), the inner surface of the PHP (Spectrum 45) and spectrum from the whole image (Map Sum Spectrum).

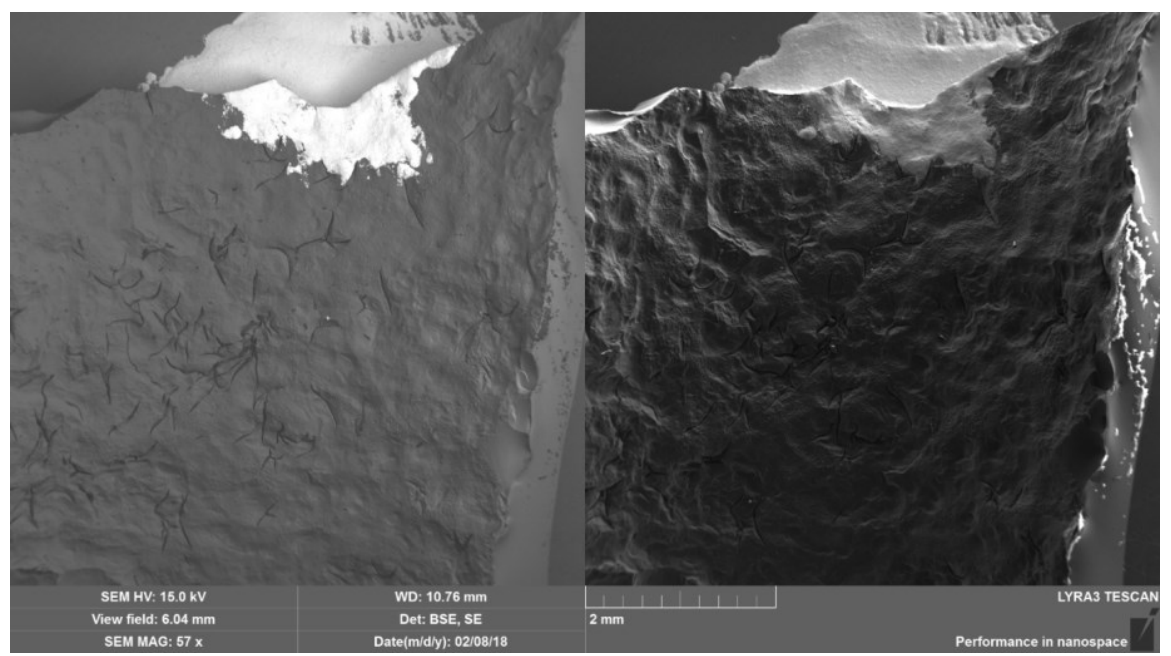

a)

b)

Fig. 13. Sample 4 - The inner material of the PHP. a) BSE image, b) SE image.

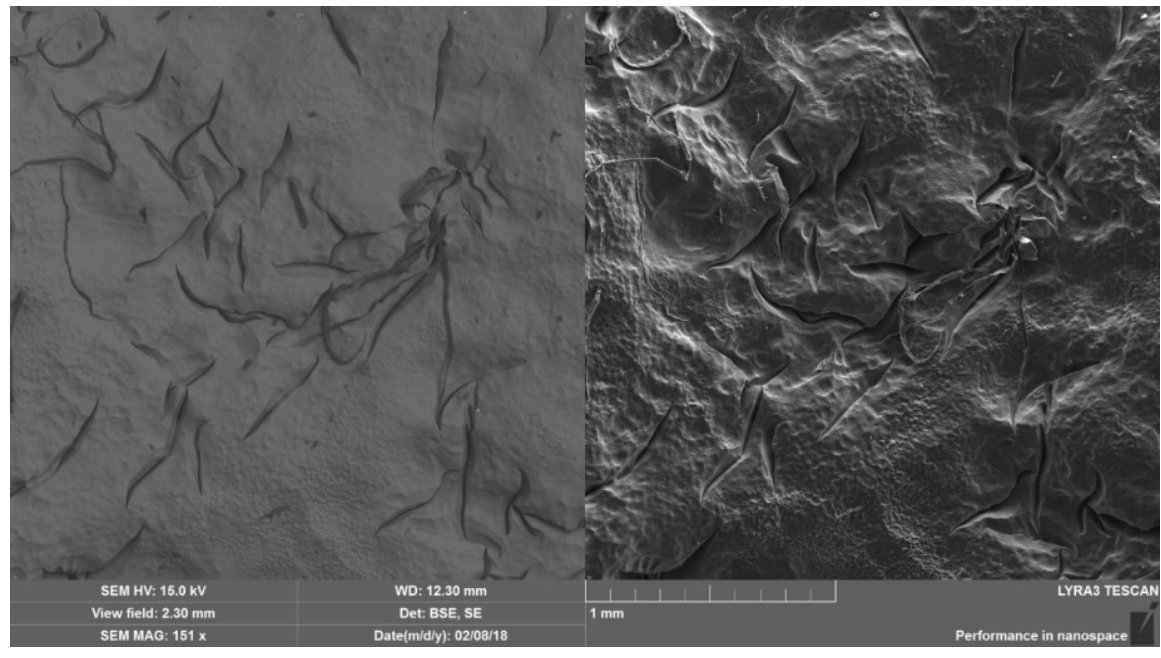

a)

b)

Fig. 14. Sample 4 - Crack detail. a) BSE image, b) SE image. 
Table 2. Chemical composition of pipe wall, EDS analysis.

\begin{tabular}{|c|c|c|c|c|c|c|}
\hline & \multicolumn{3}{|c|}{ Uncovered surface of PHP } & \multicolumn{3}{c|}{ Sodium-exposed surface of PHP } \\
\hline & O (At. \%) & $\mathrm{Si}$ (At. \%) & $\mathrm{Na}$ (At. \%) & O (At. \%) & $\mathrm{Si}$ (At. \%) & $\mathrm{Na}$ (At. \%) \\
\hline Sample 1 & 64.2 & 35.6 & 0.2 & 68.6 & 31.4 & 0.0 \\
\hline Sample 2 & 64.2 & 35.0 & 0.8 & 64.0 & 35.7 & 0.3 \\
\hline Sample 3 & 54.4 & 24.5 & 21.1 & 60.2 & 38.6 & 1.2 \\
\hline
\end{tabular}

Results from XPS and EDS analyses for samples 1 - 3 suggest formation of a sodiumenriched surface layer and broken silicon, oxygen stoichiometry. The structure of PHP is more fragile with increasing amount of sodium in the glass tube.

The sodium contamination of samples 1 and 2 was lower than 1 at.\% and the sodiumexposed surface was smooth and free of deep cracks. The cracks observed were probably caused by excessive load at a time of explosion.

The sodium contamination of sample 3 is significantly higher and deeper compared to contamination of samples 1 and 2. The topmost sodium-exposed layer contains 21 at.\% of sodium while the deepest region uncovered contains 1.2 at.\% of sodium. As displayed in Figures 9-12, the sodium attack is highly localized by forming sodium-rich fibers (1723 at.\% Na) distributed in a silicon-rich (4-5 at.\% Na) matrix. Such fibers form diffusion channels for further sodium contamination and act as vitrification and crack initiators.

Sample 4 exhibits the surface morphology completely different from samples $1-3$. The sample is characterized by a rough surface full of deep cracks. (Note: Chemical analyses were not performed due to inappropriate sample sizes - the raised parts of the tube removed the signal for the EDS).

\section{Conclusions}

Based on the different temperatures in the PHP, it can be expected that samples 1 and 2 were particularly stressed by sodium vapour, which might explain the low sodium contamination of the pipe material. The colour transition from dim white to dark brown can be probably explained by an increasing contamination of the inner surface, where sodium replaced the silicon in the oxide structure. With an increasing distance from the molten sodium level, a lower temperature and lower sodium vapour pressure can be expected.

The black surface of sample 4 can be explained as a very intensive sodium attack on the amorphous $\mathrm{SiO}_{2}$ structure by a molten sodium. The thermal effect gradient on the devitrification process shall be considered. Sample 4 was localized in the heated part of the experimental apparatus. In this area, heat is conducted through the wall in the molten sodium at higher temperatures, whereas samples $1-3$ were localized in cooled part of the experimental apparatus where the thermal gradient is in opposite direction and temperatures are significantly lower.

The pipe wall thinning can be expected due to the chemical reaction of molten sodium with the PHP's wall. The pipe was not resistant to further mechanical stress caused by operating pressure thermal gradients.

The acknowledgement: This article is supported by the financial assistance of the project KEGA No. 042ŽU-4/2016 "Cooling by physical and chemical processes".

\section{References}

1. R. Nosek, et al., AIP Conference Proceedings, 1917, 030001 (2017) 
2. M. Jobb, L. Kosa, M. Holubcik, R. Nosek, Applied Mechanics \& Materials, 832, 184191, (2016)

3. J. Krätsmár-Šmogrovič et al. Všeobecná a anorganická chémia (in Slovak), II. edition, Osveta ISBN 8080632458 (2007)

4. K.B. Richter, Prevention of sodium diffusion through furnace tube quartz, Lehigh University-Lehigh Preserve Theses and Dissertations (1984)

5. P.F. Schmidt, Contamination-Free High Temperature Treatment of Silicon or other materials, J. Electrochem. Soc., 130 (1983)

6. S.J. Rothman, Diffusion of Alkali Ions in Vitreous, Silica, 11 J. Am. Ceram. Soc.T, 65 (1982)

7. W. Heng-yu, W. Jia-fan, A study on the devitrification of silica glass tube the diffusion furnace, Silicate Journal, 9 (1981) 